Artigo / Article

\title{
Importância da presença de granulações tóxicas para o diagnóstico hematológico de septicemia
}

\section{The investigation of the presence of toxic granulation for septicemia hematologic diagnostic}

\author{
Danielle Nazaré S. Salgado ${ }^{1}$ \\ Raimundo Gladson Carvalho ${ }^{2}$ \\ Maria de Fátima P. Oliveira ${ }^{3}$ \\ Eduardo José M. Santos ${ }^{4}$ \\ Lacy Carlos Brito Junior 5
}

\begin{abstract}
Este trabalho visou investigar a associação da coexistência da presença de granulações tóxicas com resultados de hemocultura positivas, idade dos pacientes, condições de internamento e tipos de agentes bacterianos. Foi realizada análise retrospectiva e prospectiva, cega, para a presença de granulações tóxicas em amostras sangüíneas de trezentos pacientes, de ambos os sexos, internados em hospitais da Cidade de BelémPará, com solicitação de hemocultura, num período de dois anos. Com os hemogramas e as hemoculturas realizadas por métodos de automação, e todos os dados submetidos à metodologia de comparação estatística pelo Qui-quadrado (método de clump). Nossos resultados mostraram a existência de associação estatística entre: (1) a presença de granulações tóxicas e os resultados de hemoculturas positivas; (2) a menor idade dos pacientes (neonatos) associadas a hemocultura positiva; (3) a condição de internamento em UTI com hemocultura positiva; e (4) a presença de granulações tóxicas e a observação de leucocitose e desvio à esquerda, em pacientes internados em UTI, com hemoculturas positivas. E que os cinco principais agentes bacterianos identificados nas hemoculturas deste estudo foram Klebsiella oxytoca (22\%), Acinetobacter calcoaceticus (20\%), Escherichia coli (18\%), Enterobacter cloacae (14\%), e Pseudomonas aeruginosa (8\%). Rev. bras. hematol. hemoter. 2007;29(4):373-377.
\end{abstract}

Palavras-chave: Granulações tóxicas; hemoculturas; microbiologia; hematologia; diagnóstico.

\section{Introdução}

A septicemia representa o agravamento clínico-patológico da disseminação de um agente agressor, freqüentemente bacteriano, no sangue, e que pode ser diagnosticado pelo exame de hemocultura. Esta condição patológica, septicemia, aumenta os riscos de morbidade e mortalidade para o paciente, o que pode ser determinado por vários fatores, intrínsecos e extrínsecos, dentre os quais podemos destacar: as características do patógeno e a resistência deste a antibioticoterapia; o estado nutricional do paciente; e o grau e a natureza da resposta inflamatória do hospedeiro. . $-8,10,12,14,15,16^{-10}$

Nestas condições, as bactérias mais freqüentemente isoladas de hemoculturas de pacientes com septicemia são: S. aureus e Staphylococcus coagulase-negativos, S. viridans, S. pneumoniae, Enterococcus, Escherichia. coli, Klebsiella, Pseudomonas, Enterobacter, Proteus, Salmonella e

\footnotetext{
${ }^{1}$ Biomédica, funcionária do Setor de Hematologia do Laboratório de Patologia Clínica Dr. Paulo C. Azevedo.

${ }^{2}$ Graduando em Biologia, técnico em Microbiologia, funcionário do Setor de Hematologia do Laboratório de Patologia Clínica Dr. Paulo C. Azevedo. ${ }^{3}$ Graduanda em Farmácia, técnica em Hematologia, funcionária do Setor de Hematologia do Laboratório de Patologia Clínica Dr. Paulo C. Azevedo. ${ }^{4}$ Professor doutor adjunto III do Departamento de Fisiologia do Centro de Ciências Biológicas da Universidade Federal do Pará. ${ }^{5}$ Professor doutor adjunto II do Departamento de Patologia do Centro de Ciências Biológicas da UFPA. Responsável técnico pelo Laboratório de Patologia Geral, Imunopatologia e Citologia da UFPA.

${ }^{1}$ Trabalho desenvolvido no Laboratório de Patologia Geral, Imunopatologia e Citologia do Centro de Ciências Biológicas da UFPA em parceria com o Laboratório de Patologia Clínica Dr. Paulo C. Azevedo.

Correspondência: Lacy Cardoso de Brito Junior

Universidade Federal do Pará, Centro de Ciências Biológicas, Depto. de Patologia, Laboratório de Patologia Geral, Imunopatologia e Citologia Av. Augusto Corrêa $n^{\circ} 01$ - Bairro Guamá

66075-900 - Belém-PA - Brasil

Tel.: (091) 32017565; E-mail: lcdbrito@ufpa.brou lcdbrito@bol.com.br
} 
Haemophilus, muitas dessas encontradas no ambiente hospitalar, flora normal ou em colonização no paciente. ${ }^{1,4-7,12}$ Por sua vez, as principais alterações hematológicas encontradas em pacientes com septicemia são a elevação da contagem de leucócitos (leucocitose) e do número de neutrófilos (neutrofilia), com ativação destes. ${ }^{2,9,10,11,13,14,18}$ Ocasionalmente o oposto também pode acontecer em relação à contagem diferencial de leucócitos, isto é, a associação de septicemia a uma contagem de neutrófilos diminuída (neutropenia), principalmente em população pediátrica. . $^{3,4,5,7,8,12,14-17}$

A produção e a ativação aumentada de neutrófilos circulantes, neutrofilia, pode resultar em lesões teciduais sérias, que em última análise podem levar a falência múltipla de órgãos. ${ }^{2-5,5,11,15,18}$ Esta ativação de neutrófilos pode ser observada através de alterações morfológicas destas células, no sangue periférico, pela presença de granulações tóxicas, vacuolização e/ou presença de corpos de Döhle. ${ }^{1,3,8,10,16,17,20}$

As granulações tóxicas são pequenas formações em grânulos que aparecem no citoplasma dos neutrófilos e refletem uma perturbação da maturação dos mesmos, com persistência dos grânulos azurófilos nos estádios celulares maduros, ou podem ainda ser o resultado da endocitose de agentes tóxicos (bactérias, proteínas séricas desnaturadas) com formação de novos grânulos anormais. O termo "tóxico" é usado para indicar o estado de funcionamento de muitas células, que ocorre numa variedade de doenças como infecções sistêmicas, câncer, pneumonia, coma diabético ou hepático, toxemia da gravidez, envenenamento químico e em estados tóxicos. ${ }^{2,4,8,9,10,13,15,18}$

Neste contexto, este trabalho buscou avaliar as associações entre a presença de granulações tóxicas e os resultados de hemoculturas positivas ou negativas; existência de relação entre o número de cruzes de granulações tóxicas e hemoculturas positivas; entre a idade dos pacientes e hemoculturas positivas; além da associação entre a condição de internamento (UTI e outros) e hemocultura positiva; e a associação entre a presença de granulações tóxicas com leucocitose, neutrofilia, desvio à esquerda, anemia e plaquetopenia em pacientes internados em hospitais.

\section{Casuística e Métodos}

\section{Casuística}

Foi realizado estudo retrospectivo e prospectivo para a presença de granulações tóxicas em amostras sangüíneas de trezentos pacientes, de ambos os sexos, internados em hospitais da Cidade de Belém - PA, com atendimento laboratorial no Laboratório de Patologia Clínica, após solicitação médica de hemocultura e hemograma, no período de setembro de 2001 a setembro de 2003. Os parâmetros avaliados foram dados clínicos como idade, condição de internamento (UTI e outros) e o número de cruzes de granulações tóxicas associados à hemocultura positiva de cada paciente.
Análise do hemograma e da morfologia de neutrófilos

Os hemogramas de todos os pacientes foram realizados no Laboratório, através de metodologia de automação, por meio do Contador Automático MAXM Coulter, e da observação da lâmina de todos os pacientes que tinham solicitação de hemocultura, para a verificação da presença de granulações tóxicas em neutrófílos. Além da análise dos seguintes parâmetros nestes pacientes: presença de leucocitose, neutrofilia, desvio à esquerda, anemia e plaquetopenia. O controle na identificação da presença de granulações tóxicas nos hemogramas selecionados foi realizado por análise cega do material, estudo prospectivo, por três observadores diferentes, sendo considerada positiva a presença de granulações tóxicas (GT) quando houvesse concordância entre todos os observadores, ou através de estudo retrospectivo de laudos já prontos. A classificação da quantidade de granulações tóxicas em neutrófilos foi definida da seguinte maneira: (Zero) granulação normal; $(+)$ leve; $(++)$ aproximadamente $50 \%$ dos neutrófilos contendo grânulos escuros; $(+++)$ intensa granulação na maioria das células; e (++++) GT grosseiras, com o núcleo dos neutrófilos sendo obscurecido por elas.

\section{Hemocultura}

As hemoculturas de todos os pacientes hospitalizados foram realizadas no Laboratório, através de metodologia de automação, com o equipamento VITEK Bactec 9120 para estas análises, e a partir de procedimentos bacteriológicos tradicionais para a identificação dos agentes envolvidos quando da presença de hemocultura positiva.

\section{Análise estatística}

Todos os dados de hemograma, hemocultura e clínicos foram submetidos a tratamento estatístico para a determinação da associação da presença de granulações tóxicas com a presença de hemocultura positiva para cada paciente. Para tanto, estes dados foram submetidos à metodologia de comparação estatística pelo Qui-quadrado (método de clump) (Sham \& Curtis, 1995).

\section{Resultados e Discussão}

A análise dos resultados de hemocultura associados à presença ou ausência de granulações tóxicas em hemogramas de pacientes, que tiveram seus exames realizados no Laboratório de Patologia Clínica, e que estavam internados em hospitais da cidade de Belém (Gráfico 1 e Tabela 1), sugere que existe associação $(p<0.05)$ entre a presença de granulações tóxicas e os resultados de hemoculturas positivos, com 73\% dos pacientes que apresentavam granulações tóxicas também com hemoculturas positivas. Assim, nossos dados sugerem que a ausência de granulações tóxicas pode ser considerada um fator excludente para a presença de septicemia, visto que a maioria dos pacientes com hemoculturas negativas também não apresentava granulações tóxicas (60\% dos casos). 
Tabela 1. Associação dos resultados de hemoculturas e presença / ausência de granulações tóxicas

\begin{tabular}{cccc}
\hline $\begin{array}{c}\text { Granulações/ } \\
\text { Hemocultura }\end{array}$ & $\begin{array}{c}\text { Presença de } \\
\text { granulações } \\
\text { tóxicas }\end{array}$ & $\begin{array}{c}\text { Ausência de } \\
\text { granulações } \\
\text { tóxicas }\end{array}$ & Total \\
\hline $\begin{array}{c}\text { Hemocultura } \\
\text { positiva } \\
\text { Hemocultura } \\
\text { negativa }\end{array}$ & $95(73 \%)$ & $35(27 \%)$ & $130(100 \%)$ \\
\hline Total & $164(40 \%)$ & $101(60 \%)$ & $170(100 \%)$ \\
\hline $\mathrm{p}<0.05$ & & $136(45 \%)$ & $300(100 \%)$ \\
\hline
\end{tabular}

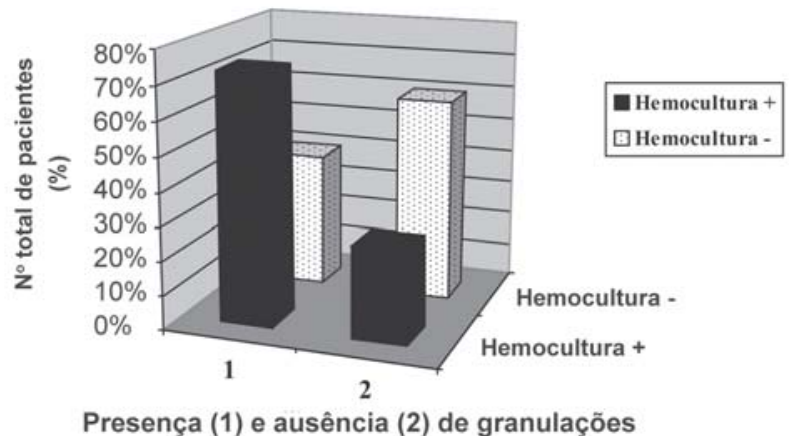

Gráfico 1. Associação entre resultados de hemoculturas e presença de granulações tóxicas

Quando avaliamos o número de cruzes de granulação tóxica em neutrófilos e o resultado das hemoculturas (Gráfico 2 e na Tabela 2) em pacientes que realizaram, concomitantemente, hemograma e hemocultura, 164 pacientes, observamos que estes dados não revelaram significância estatística para a associação da presença de hemoculturas positivas com o maior ou menor número de cruzes de granulação tóxica em neutrófilos ( $\mathrm{p}>0.05$ ), demonstrando que o número de cruzes não é uma informação fundamental para a determinação da presença de septicemia.

Como apresentado na Tabela 2 e Gráfico 2, por sua vez, o aumento dos percentuais de granulações tóxicas em pacientes com hemocultura negativa pode estar relacionado ao fato de que, no momento da colheita do material, alguns pacientes já estivessem fazendo uso de antibioticoterapia, prática médica comum em função da gravidade de alguns pacientes, o que pode interferir especificamente nas condições morfológicas de neutrófilos, independente da presença de agente agressor. Dados corroborados com a literatura. ${ }^{3-7,12,14,16,17}$

Clinicamente, porém, é sabido que não é possível, apenas com os resultados de presença ou ausência de granulações tóxicas, associarem-se estes dados a uma suspeita de septicemia, sendo necessário também a associação destas granulações tóxicas a outras alterações hematológicas no hemograma, como a presença de leucocitose, neutrofilia, desvio à esquerda (D.E.), plaquetopenia e anemia. Assim, neste estudo nós também submetemos estes dados (Gráfico 3 e
Tabela 2. Associação entre $n^{0}$ de cruzes de granulação tóxica e resultados de hemoculturas

\begin{tabular}{cccc}
\hline $\begin{array}{c}N^{0} \text { de Cruzes } \\
\text { de Granulação } \\
\text { Tóxica }\end{array}$ & $\begin{array}{c}\mathrm{N}^{0} \text { de } \\
\text { Hemoculturas } \\
\text { Positivas }\end{array}$ & $\begin{array}{c}\mathrm{N}^{\circ} \text { de } \\
\text { Hemoculturas } \\
\text { Negativas }\end{array}$ & Total \\
\hline+ & $34(43 \%)$ & $45(57 \%)$ & $79(100 \%)$ \\
++ & $24(37 \%)$ & $40(63 \%)$ & $64(100 \%)$ \\
+++ & $7(33 \%)$ & $14(67 \%)$ & $21(100 \%)$ \\
\hline Total & $65(39 \%)$ & $99(61 \%)$ & $164(100 \%)$ \\
\hline
\end{tabular}

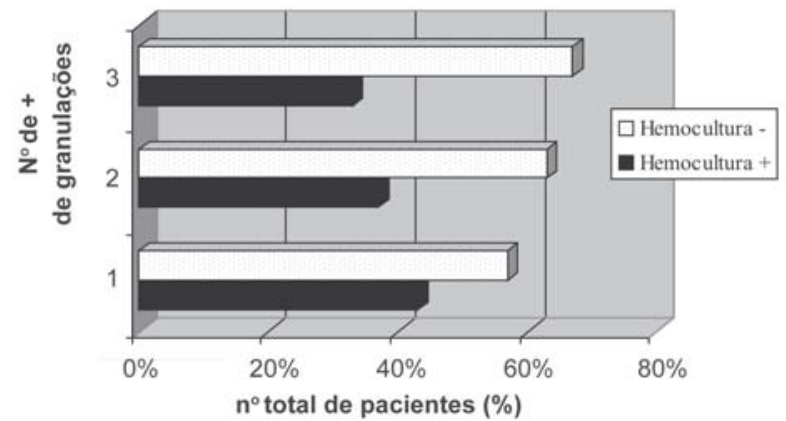

Gráfico 2. Associação entre $n^{\circ}$ de $(+)$ de granulações tóxicas e resultados de hemocultura.

Tabela 3) a uma análise estatística para verificação de sua associação com o diagnóstico de septicemia, através de comprovação por hemocultura positiva, a qual demonstrou significância estatística para os achados de leucocitose (70\%) e desvio à esquerda (68\%) com hemoculturas positivas, dados corroborados por outros autores na literatura. ${ }^{1-5,8,9,10,13,14,15,18,19}$ Com esta análise tendo sido submetida a uma correção estatística, onde P foi multiplicado por cinco (o que corresponde ao número de categorias analisadas).

Quando, por sua vez, investigamos a associação entre pacientes internados em UTI e a positividade de suas respectivas hemoculturas (Gráfico 4 e Tabela 4), observamos que, nos pacientes internados em UTI, as porcenta-

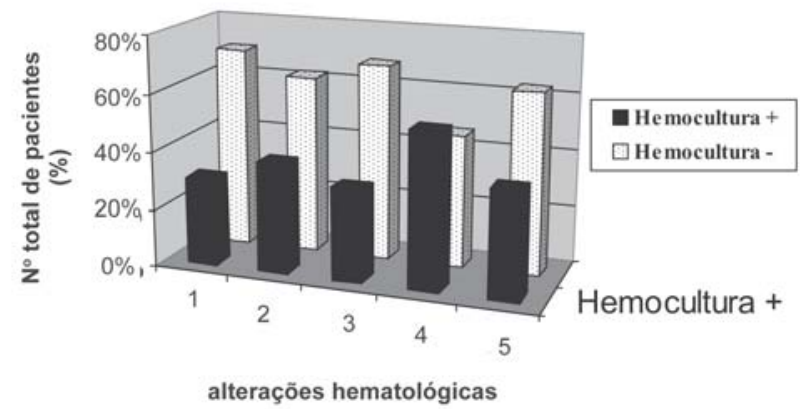

Gráfico 3. Associação de alterações hematológicas e septicemia. A numeração indica as seguintes alterações: (1) leucocitose. (2) neutrofilia, (3) desvio à esquerda, (4) plaquetopenia, e (5) anemia 
Tabela 3. Associação entre alterações hematológicas e septicemia

\begin{tabular}{cccccc}
\hline $\begin{array}{c}\text { Alterações } \\
\text { hematológicas/ } \\
\text { Hemocultura }\end{array}$ & Leucocitose* & Neutrofilia & $\begin{array}{c}\text { Desvio à } \\
\text { esquerda }\end{array}$ & Plaquetopenia & Anemia \\
$\begin{array}{c}\text { Hemocultura } \\
\text { positiva }\end{array}$ & $80(70 \%)$ & $59(62 \%)$ & $82(68 \%)$ & $31(46 \%)$ & $48(63 \%)$ \\
$\begin{array}{c}\text { Hemocultura } \\
\text { negativa }\end{array}$ & $34(30 \%)$ & $36(38 \%)$ & $39(32 \%)$ & $36(54 \%)$ & $28(37 \%)$ \\
\hline Total & $114(100 \%)$ & $95(100 \%)$ & $121(100 \%)$ & $67(100 \%)$ & $76(100 \%)$ \\
\hline $\mathrm{P}<0.05$ & & & & &
\end{tabular}

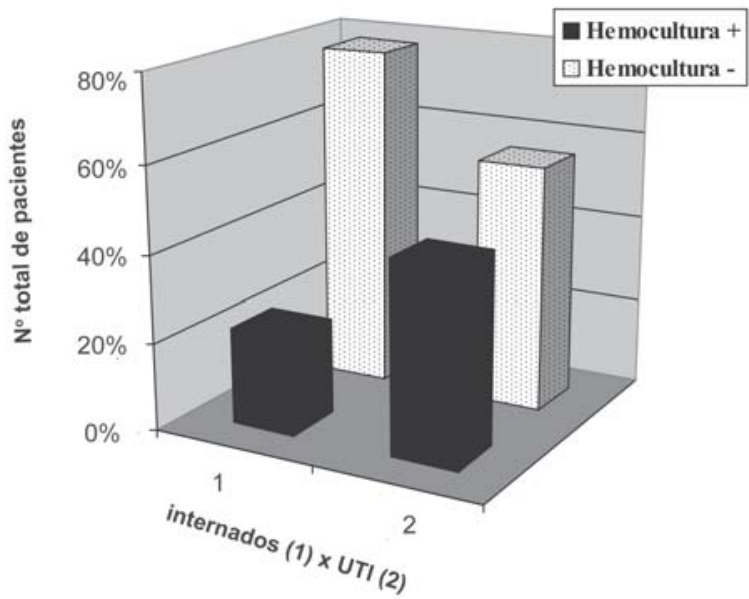

Gráfico 4. Associação entre pacientes em UTI e hemoculturas positivas

Tabela 4. Associação entre pacientes em UTI e hemoculturas positivas

\begin{tabular}{cccc}
\hline $\begin{array}{c}\text { Pacientes/ } \\
\text { Hemocultura }\end{array}$ & Hemocultura + & Hemocultura - & Total \\
\hline $\begin{array}{c}\text { Pacientes } \\
\text { internados } \\
\begin{array}{c}\text { Pacientes } \\
\text { em UTI }\end{array}\end{array}$ & $7(22 \%)$ & $25(78 \%)$ & $32(100 \%)$ \\
\hline Total & $65(46 \%)$ & $99(54 \%)$ & $164(100 \%)$ \\
\hline $\mathrm{P}<0.05$ & &
\end{tabular}

gens de hemoculturas positivas foi bem maior (44\%) que os resultados de hemoculturas positivas de pacientes internados em outras condições (22\%), como enfermarias ou apartamentos, demonstrando que o encaminhamento clínico de pacientes para UTI, com suspeitas de septicemias, está sendo realizado de forma adequada $(\mathrm{p}<0.05)$.

A verificação da presença de hemoculturas positivas em pacientes não neonatos e pacientes neonatos (Gráfico 5 e Tabela 5), em nosso estudo, demonstrou que a porcentagem de hemoculturas positivas em pacientes não neonatos foi bem inferior (25\%) que a porcentagem de hemoculturas positivas em pacientes neonatos (56\%). Mostrando que existe uma associação, de significância estatística $(\mathrm{p}<0.0005)$ entre pacientes neonatos e hemoculturas positivas, e que susten-

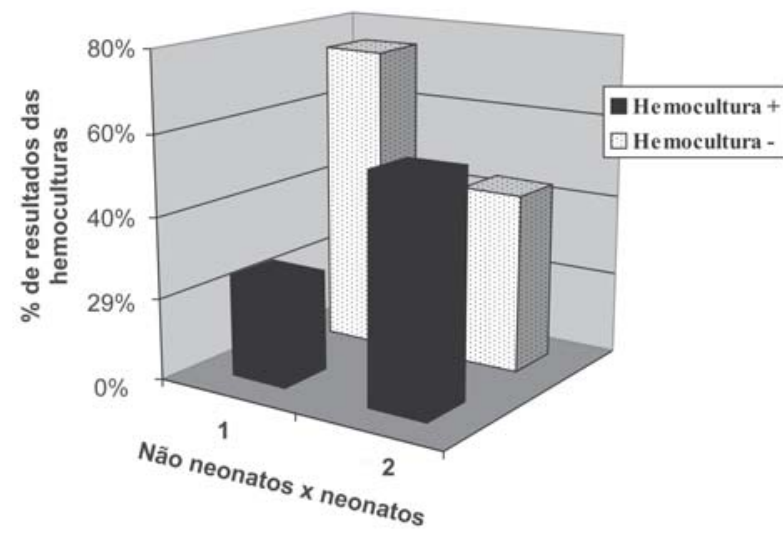

Gráfico 5. Associação entre pacientes neonatos e hemoculturas positivas.

Tabela 5 Associação entre pacientes neonatos e hemoculturas positivas

\begin{tabular}{cccc}
\hline $\begin{array}{c}\text { Paciente/ } \\
\text { Hemocultura }\end{array}$ & $\begin{array}{c}\text { Hemocultura } \\
\text { Positiva }\end{array}$ & $\begin{array}{c}\text { Hemocultura } \\
\text { Negativa }\end{array}$ & Total \\
\hline $\begin{array}{c}\text { Pacientes não } \\
\text { neonatos } \\
\begin{array}{c}\text { Pacientes } \\
\text { neonatos }\end{array}\end{array}$ & $21(25 \%)$ & $64(75 \%)$ & $85(100 \%)$ \\
\hline Total & $65(46 \%)$ & $35(44 \%)$ & $79(100 \%)$ \\
\hline $\mathrm{P}<0.05$ & $99(60 \%)$ & $164(100 \%)$ \\
\hline
\end{tabular}

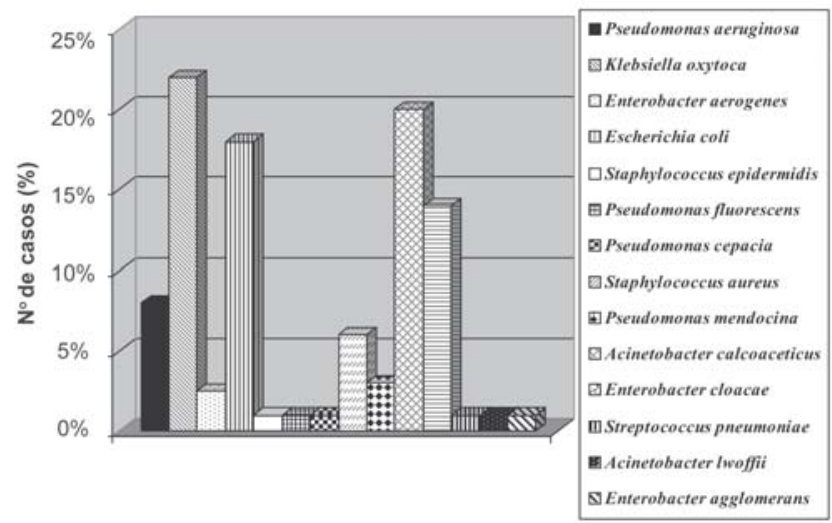

Gráfico 6. Bactérias identificadas nas hemoculturas positivas

ta a nossa hipótese de que a antibioticoterapia é uma conduta clínica prévia, antes da coleta dos exames, principalmente por se tratarem de pacientes neonatos onde a conduta clínica tem que ser rápida em função da alta morbidade e letalidade destes pacientes. . $^{1,3-7,12,15,16,17}$

Como dado ilustrativo do nosso trabalho foi observado ainda que os agentes bacterianos de maior prevalência (Gráfico 6), em hemoculturas positivas (77 casos), foram as bactérias Klebsiella oxytoca (22\%), Acinetobacter calcoaceticus (20\%), Escherichia coli (18\%), Enterobacter cloacae (14\%), Pseudomonas aeruginosa (8\%). Dados compatíveis com a literatura. ${ }^{1,3-7}$ 


\section{Conclusão}

Nossos resultados mostram existir uma associação entre a presença de granulações tóxicas e a observação de leucocitose e desvio à esquerda em hemogramas de um mesmo paciente (neonatos) internado em hospital, principalmente em UTI, com hemoculturas positivas. Mostra ainda que não existe relevância estatística na associação entre o número de cruzes de granulações tóxicas (grau de evolução) e a positividade da hemocultura.

\section{Agradecimentos}

Agradecemos cordialmente ao Dr. Paulo Sergio Roffe Azevedo e a todos do Laboratório de Patologia Clínica Dr. Paulo C. Azevedo pelo apoio logístico e técnico dado para o desenvolvimento deste trabalho.

\begin{abstract}
This work aims at investigating the association of the presence of toxic granulation with positive blood cultures, age of patients, conditions of hospitalization and types of bacterial agents. Blind prospective and retrospective, analyses were carried out for the presence of toxic granulations-in blood samples of 300 patients of the both genders hospitalized in the City of Belém, Pará, Brazil. Request blood tests over a two year period were evaluated. The blood tests and cultures were performes using automated methods. All the data were statistically compared using the Qui-square test (clump method). The results show statistical associations between: (1) the presence of toxic granulations and positive blood cultures; (2) lower ages of patients (the newborn) and positive blood cultures; (3) hospitalization in the ICU and positive blood cultures and (4) toxic granulations-and the observation of leucocytosis and right-left shunts in patients hospitalized in the ICU with positive blood cultures. The commonest bacterial agents identified were klebsiella oxytoca (22\%), Acinetobacter calcoaceticus (20\%), Escherichia coli (18\%), Enterobacter cloacae (14\%), and Pseudomonas aeruginosa (8\%). Rev. bras. hematol. hemoter. 2007;29(4):373-377.
\end{abstract}

Key words: Toxic granulations; hemocultures; microbiology; hematology; diagnostic.

\section{Referências Bibliográficas}

1. Aird Wc. The hematologic system as a marker of organ disfunction in sepsis. Mayo Clinical Procedures. 2003;78:869-81.

2. Bernard J, Levy JP, Vareti B, Clauvel JP, Rain JD, Sultan, Y. Hematologia, São Paulo, Editora Medsi. 300p. 2000.

3. Bernstein HM, Pollock BH, Calhon DA, Christensen RD. Administration of recombinant granulocyte colony-stimulating factor to neonates with septicemia: a meta-analysis. Journal of Pedriatrics. 2001;138:917-20.

4. Funke A, Berner R, Traichel B, Schmeisser D, Leititis JU, Niemeyer $\mathrm{CM}$. Frequency, natural course, and outcome of neonatal neutropenia. Pediatrics. 2000;106(1):45-51.

5. Gawaz M, Dickfeld T, Bogner C, Fateh-Moghadam S, Neumann FJ. Platelet function in septic multiple organ dysfunction syndrome. Intensive Care Medical. 1997;23:379-85.
6. Gaynes RP, Edwards JR, Jarvis WR, Culver DH, Tolson JS, Martone WJ. Nosocomial infections among neonates in high-risk nurseries in the United States. National Nosocomial Infections Surveillance System. Pediatrics. 1996;98:357-61.

7. Gray JE, Richardson DK, Mccormick MC, Goldmann DA. Coagulase-negative staphylococcal bacteremia among very low birth weight infants: relation to admission illness severity, resource use, and outcome. Pediatrics. 1995;95:225-30.

8. Held TK, Cross AS. Role of hematopoietic growth factors in nonneutropenic infections in sepsis. Current Opinion in Hematology. 1999;6:176-83.

9. Heller AR, Groth G, Heller SC. N-acetylcystein reduces respiratory burst but augments neutrophil phagocytosis in intensive care unit patients. Critical Care Medicine. 2002;29:272-6.

10. Hillman RS, Ault KA. Hematology in Clinical Practice, McGrawHill Companies. p.630. 2002.

11. Jaeschke H, Ho YS, Fisher MA, Lawson JA. Glutathione peroxidasedeficient mice are more susceptible to neutrophil-mediated hepatic parenchymal cell injury during endotoxemia: importance of an intracellular oxidant stress. Hepatology. 1999;29:443-50.

12. Marshall JC. Sirs And Mods: what is their relevance to the science and practice of intensive care? Shock. 2000;14(6):586-9.

13. Nieuwland R, Berckmans RJ, Mcgregor S. Cellular origin and procoagulant properties of microparticles in meningococcal sepsis, Blood. 2000;95:930-5.

14. Root RK, Dale DC. Granulocyte colony-stimulating factor and granulocyte-macrophage colony stimulating factor: comparisons and potential for the use in the treatment of infections in nonneutropenic Patients. Journal Infectious Disease. 1999;179(2): 342-52.

15. Sham PC, Curtis D. Monte Carlo tests for associations between disease and alleles at highly polymorphic loci. Annals of Human Genetics. 1995;59:97-105.

16. Shoupe M, Weisenberger JM, Wang JL, Pyle JM, Gamelli RL, Shankar R. Mechanisms of neutropenia envolving myeloid maturation arrest in burn sepsis. Annals of Surgery. 1998;228:112-22.

17. Stoll BJ, Gordon T, Korones SB. Early-onset sepsis in very low birth weight neonates: a report from the National Institute of Child Health and Human Development Neonatal Research Network. Journal of Pediatrics. 1996;129:72-80.

18. Stoll BJ, Gordon T, Korones SB. Late-onset sepsis in very low birth weight neonates: a report from the National Institute of Child Health and Human Development Neonatal Research Network, Journal of Pediatrics. 1996b;129:63-71.

19. Tillema MS, Lorenz KL, Weiss MG, Dries DJ. Sublethal endotoxemia promoters pulmonary cytokine-induced neutrophil chemoattractant expression and neutrophil recruitment but not over lung injury in neonatal rats. Biology of Neonatals. 2000;78:308-14.

20. Zahorec R. Ratio of neutrophil to lymphocyte cause - rapid and simple parameter of systemic inflammation and distress in critically ill. Bratisl Lek Listy. 2001;102:5-14.

21. Zucker-Franklin D. The phagosomes in rheumatoid synovial fluid leukocytes: a light, fluorescence, and electron microscope study. Arthritis Rheumatoides. 1996;9(1):24-36.

Avaliação: Editor e dois revisores externos

Conflito de interesse: não declarado

Recebido: 25/08/2006

Aceito após modificações: 05/04/2007 